\title{
Development of a Screw-Crane System for Pre-Lifting the Sternal Depression in Pectus Excavatum Repair: A Test of Mechanical Properties for the Feasibility of a New Concept
}

\author{
Hyung Joo Park, M.D., Ph.D., Gongmin Rim, M.D. \\ Department of Thoracic and Cardiovascular Surgery, Seoul St. Mary's Hospital, College of Medicine, The Catholic University of Korea, Seoul, Korea
}

\section{ARTICLE INFO}

Received January 21, 2021

Revised March 15, 2021

Accepted March 21, 2021

Corresponding author

Hyung Joo Park

Tel $82-2-2258-2858$

Fax 82-2-594-8644

E-mail hyjpark@catholic.ac.kr

ORCID

https://orcid.org/0000-0003-0886-0817
Background: Pre-lifting of the sternum marked a major turning point in pectus excavatum repair. The author developed the crane technique in 2002 and successfully applied it to more than 2,000 cases using sternal wire stitching. However, blind sternal suturing limited the use of the wire-stitch crane. We propose a novel screw for sternal lifting as a new tool for the crane technique.

Methods: We developed a screw system strong enough to withstand the pressure needed for sternum lifting. The screw was designed to have a broader thread to hold the bony tissue securely. The screw's sustaining power was tested using the torsion, driving torque, and axial pull-out tests in a polyurethane block and ex-vivo porcine sternum.

Results: The screws were easily driven into the sternum, and the head of the screw was connectable to the table-mounted retractor. In the torsion test, the $2^{\circ}$ offset torsional yield was $4.53 \mathrm{~N} \cdot \mathrm{m}$ (reference value, $1 \mathrm{~N} \cdot \mathrm{m}$ ). In the polyurethane block driving torque test, the maximum torque was $0.98 \mathrm{~N} \cdot \mathrm{m}$ (reference value, $0.70 \mathrm{~N} \cdot \mathrm{m}$ ). The axial pull-out test was 446 $\mathrm{N}$ (reference value, $100 \mathrm{~N}$ ). The maximum pull-out resistance in the ex-vivo porcine sternum model was 1,516 N.

Conclusion: The screw crane was strong enough to sustain the chest wall weight to be lifted. Thus, the screws could effectively replace the sternal wire stitching in crane pre-lifting of the sternum. We expect that application of the screw-crane will be easy and that it will improve the safety and success rate of pectus repair surgery.

Keywords: Pectus excavatum, Screw, Minimally invasive repair, Sternal pre-lifting, Crane

\section{Introduction}

Crane lifting of the depressed sternum has become popular in pectus excavatum repair owing to its advantages $[1,2]$; sternal elevation provides a space between the heart and depressed sternum/chest wall and reduces the chest wall pressure (Fig. 1).

Since 2002, we have been applying the crane technique through sternal stitching; the lower tip of the sternum, where the bottom of the pectus excavatum forms, is lifted with a wire suture. The wire suture is then moved to the tip or the lateral side of the sternal body to meet more targets for repair [1]. We have found sternal stitching to be the most convenient and safe way to install a crane, and we have successfully performed over 2,000 procedures over a
A

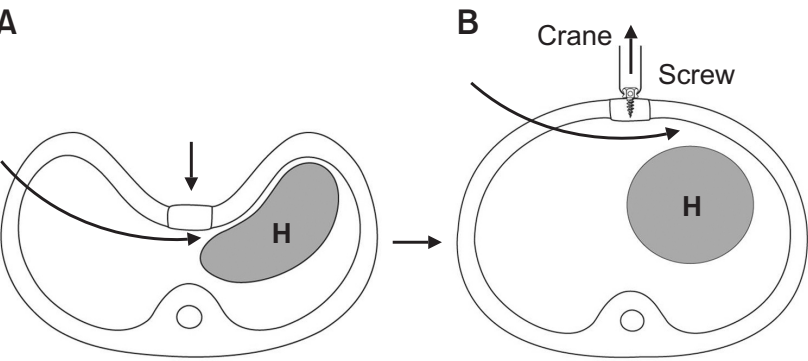

Fig. 1. Changes in chest wall and internal organ positions in preand post-crane states. (A) Pre-crane status of the pectus excavatum: there is no space between the heart $(\mathrm{H})$ and the depressed chest wall. The access route for mediastinal passage is tight (curved arrow). (B) Post-crane status with the screw-crane apparatus: the chest wall is fully elevated and there is ample space between the heart $(\mathrm{H})$ and the chest wall. The access route for mediastinal passage is wide open (curved arrow). 
period of 18 years with no complications.

Despite its advantages, many surgeons have felt uncomfortable with sternal stitching. For this reason, we developed the screw-crane system, aiming to simplify the technique and make it universally adaptable even by less experienced surgeons.

\section{Methods}

Stainless steel screws were made in different sizes to match variability in sternal thickness (Fig. 2). The threads of the screw were large enough to bear the sternal weight. The head of the screw was designed to be connected to the overhung sidebar of the retractor with a wire loop (Fig. 3).

The metallic bone screw strength was tested with the torsion test, the driving torque test, and the axial pull-out test. The test was conducted at Korea Testing Laboratory (Seoul, Korea). The tests were performed using the following ASTM F543-17 Standard specification and test methods for metallic medical bone screws: the annex 1 test method for determining the torsional properties, annex 2 for the driving torque, and annex 3 for the axial pull-out strength of metallic bone screws. The torsion test to determine the torsional properties was performed by fixing the screw on the spinning tester at a test rate of $1 \mathrm{rpm}$. The driving torque test was performed by measuring the torque while driving the screw into the solid rigid polyurethane foam (grade 40 per cubic foot) test block and spinning at a rate of $1 \mathrm{rpm}$. The axial pull-out test measured the resistance of the threads by pulling them up at a rate of $5 \mathrm{~mm} /$ min in the polyurethane foam (grade 40 per cubic foot) block. To test in a biological model, screw pulling was performed in an ex-vivo raw porcine sternum at the rate of 5 $\mathrm{mm} / \mathrm{min}$ (Fig. 4). The porcine sternum samples for the tests were procured from butchered adult pigs chosen through random selection. Four fresh porcine sternum blocks were used in the tests.

Institutional Review Board of Catholic Medical Center, The Catholic University of Korea approval was waived because the experiments were conducted on non-human objects (polyurethane blocks) and non-living animal samples (porcine sternum).

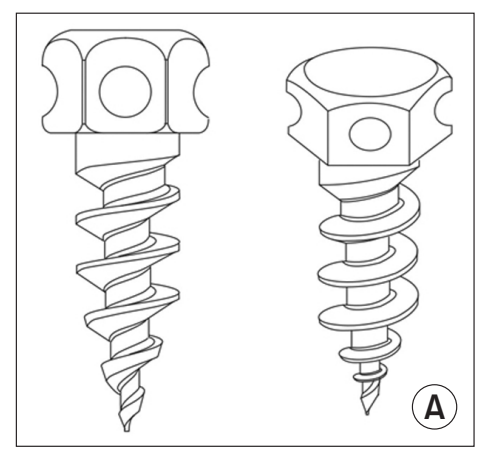

(A)

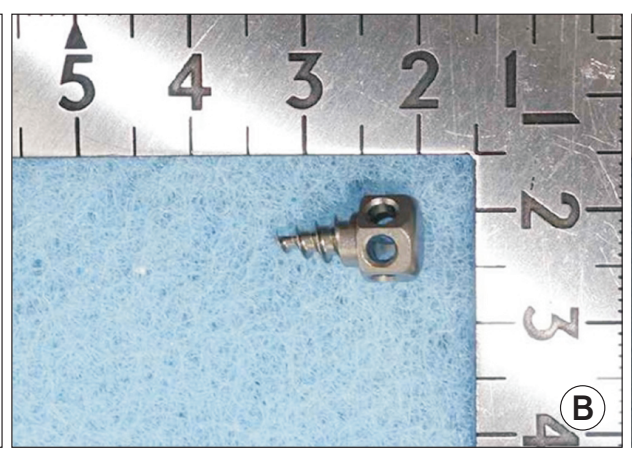

8-mm length

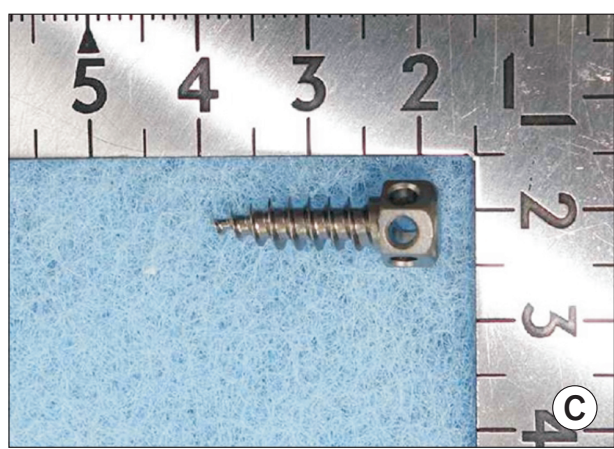

16-mm length

Fig. 2. Screws for sternal lifting: broader threads for weight tolerance (A) and different lengths for different sternal thicknesses (B, C). The head has holes for easy passage of a wire loop for connection to the overhung retractor.
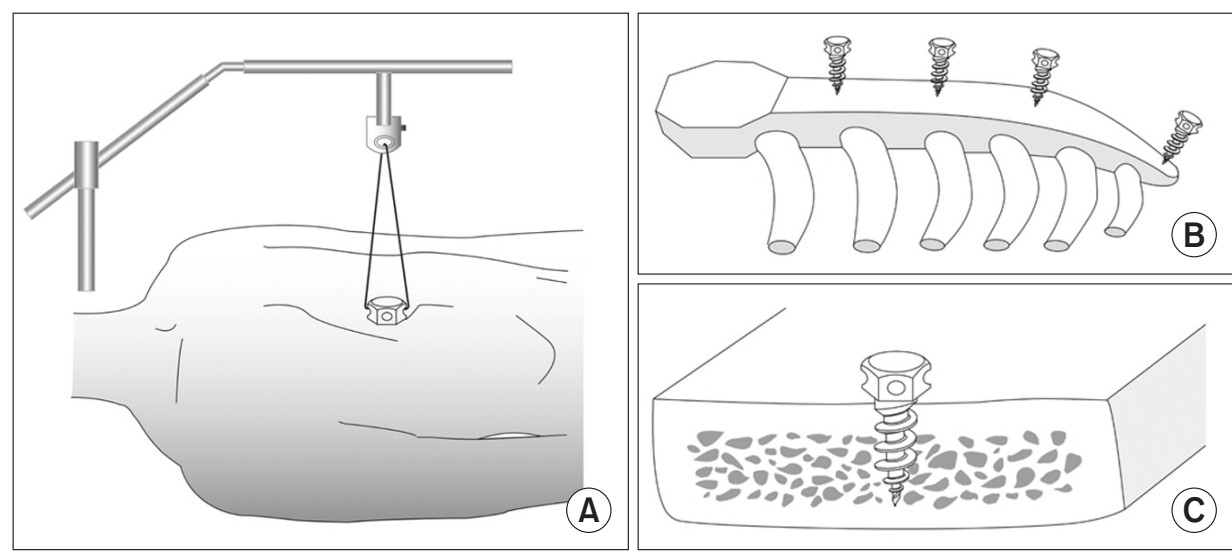

Fig. 3. Screw crane system. (A) The crane with the sternal screw is used to lift the depressed sternum. (B) The location for screw-in is liberally chosen at any part of the sternum according to the chest wall morphology. (C) The screw size is predetermined according to the sternal thickness. 


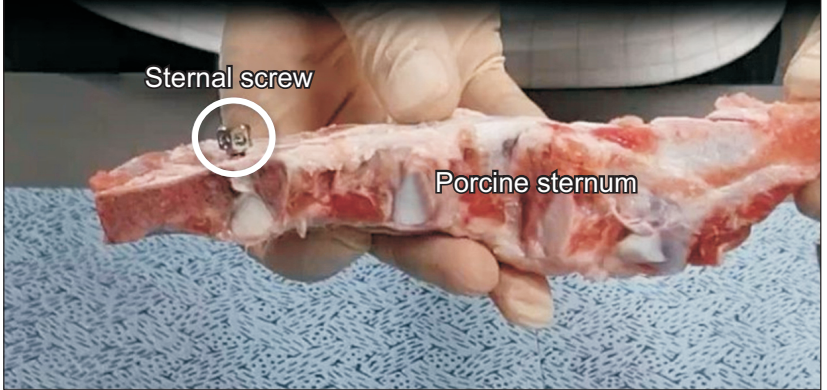

Fig. 4. Ex-vivo porcine sternal model harboring the screw in the body (circle).

\section{Results}

The screws were developed in 2 different thread diameters $(3.5 \mathrm{~mm}$ and $5 \mathrm{~mm})$ and lengths $(8 \mathrm{~mm}$ and $16 \mathrm{~mm})$ to match the pressure and the thickness of the sternum. The tips were tapered to dig easily through the outer table of the sternum (Fig. 2). The screws were easily driven into the polyurethane block and the porcine sternum. The head of the screw could accept a wire loop through the hole and was easily linked with the table-mounted retractor (the Easy Crane) (Fig. 3). The test results were as follows: torsion test with the $5-\mathrm{mm}$ screw (mean), $2^{\circ}$ offset torsional yield, $4.53 \mathrm{~N} \cdot \mathrm{m}$ (reference value, $1 \mathrm{~N} \cdot \mathrm{m}$ ); maximum torque, $5.57 \mathrm{~N} \cdot \mathrm{m}$; and breaking angle, $61^{\circ}$. The driving torque test in the solid rigid polyurethane foam block (grade 40 per cubic foot) with a pilot hole of $1.0 \mathrm{~mm}$ showed the following mean values for the $3.5-\mathrm{mm}$ screw: insertion maximum torque, $0.98 \mathrm{~N} \cdot \mathrm{m}$ (reference value, $0.70 \mathrm{~N} \cdot \mathrm{m}$ ); and removal maximum torque, $0.64 \mathrm{~N} \cdot \mathrm{m}$ (reference value, 0.50 $\mathrm{N} \cdot \mathrm{m}$ ). The mean values for the $5-\mathrm{mm}$ screw were as follows: insertion maximum torque, $0.87 \mathrm{~N} \cdot \mathrm{m}$ (reference value, 0.70 $\mathrm{N} \cdot \mathrm{m}$ ); and removal maximum torque, $0.56 \mathrm{~N} \cdot \mathrm{m}$ (reference value, $0.50 \mathrm{~N} \cdot \mathrm{m}$ ). The axial pull-out test with the $3.5-\mathrm{mm}$ screw in a $1.0-\mathrm{mm}$ pilot hole and $4.5-\mathrm{mm}$ insertion depth yielded a mean result of $446 \mathrm{~N}$ (reference value, $100 \mathrm{~N}$ ). In the ex-vivo porcine sternum model, the mean values for the 3.5-mm-diameter screw pull-out resistance were 1,241 $\mathrm{N}$ for the $8-\mathrm{mm}$-long screw and $1,516 \mathrm{~N}$ for the $16-\mathrm{mm}$ long screw.

\section{Discussion}

Pre-lifting of the sternum plays a critical role in pectus excavatum repair, before passing the introducer and bar through the mediastinum. Primarily, it provides a wider space and better visualization of the interface of the heart and the sunken chest wall for safe passage, avoiding vital organ impingement [1-3] (Fig. 1). Furthermore, once the crane fully elevates the chest wall depression, it reduces the pressure and resistance as the bar pushes the chest wall up, prevents intercostal muscle stripping, and protects the chest wall and internal organs. Holding the chest wall up with the crane until satisfactory bar fixation is achieved makes the entire repair procedure effortless and safe.

We started to use the pectus bar technique for pectus deformity repair in 1999 [4], 1 year after the Nuss technique was introduced [5]. Although it was a milestone, the technique had significant complications and failure rates, which can be attributed to 2 main issues. First, internal organ injuries that occur while passing the pectus bar through the sunken chest wall and the abutted compressed heart can cause cardiac injury with serious hemorrhage. Some patients had fatal consequences due to cardiac perforation [6]. Second, procedures failed in cases with a stiff and heavy chest wall because the system involved was not strong enough to lift the chest wall to the optimal level. The power generated by pectus bar rotation alone was insufficient to elevate the heavy adult chest wall [7]. To solve this problem, in 2002, for the first time, the primary author (H.J.P.) developed the crane technique, wherein pre-lifting of the chest wall is the first step in the pectus repair procedure. The sternum is craned up with the help of a table-mounted retractor. The retractor's sidearm over the patient's chest wall pulls the wire stitch and the sternum up [1]. The crane technique was first officially acknowledged in 2003, at the 34th American Pediatric Surgical Association Meeting and mentioned in a publication in 2004 (the conference discussion section, p. 395), which states, "I have developed another technique to prevent pericardial injury, which is the preliminary elevation of the sternum by a homemade retractor before the pectus clamp passage [7]." Since then, the crane technique has been an indispensable component of the surgical armamentarium for pectus excavatum repair and has been used successfully in more than 2,000 cases within the past 18 years.

The crane technique has evolved from simple hooking to crane-powered pectus surgery with multiple points of sternal wiring. The crane technique started without premeditation in a case with a large, heavy chest. The pectus bar failed to lift the excavated chest wall, and the case was on the verge of failure. A nick at the tip of the xiphisternum was made and a large bone hook was shoved beneath the sternum to help lift the chest wall and rescue the situation. The hook was hung over the ether screen sidebar with a wire string and the wire was rolled around a Kocher clamp 
and used to lift the sternum. The evolution of the author's crane technique has seen hook insertion, handheld lifting, and additional skin cuts being eliminated; the current technique with multiple sternal wiring using the "Easy Crane" system is handy and efficient (Fig. 5). The improved crane has a slot that fits the side rail of the operating table, and it can be slide-fixed on it during the operation at any time when necessary (Fig. 5). The multiple cranes provide powerful, consistent lifting of any location in the chest wall whenever needed throughout the procedure.

The merits of crane pre-lifting have attracted other surgeons, but their techniques seemed to be only basic and required improvement. First, manual hooking, as the most primitive technique, can lift the sternum or chest wall, but it is unstable and insufficient. It obtains some space [8,9], but only to a limited extent owing to the insufficient capacity of human lifting by a surgical assistant. As mentioned, hooking was the first author's approach before inventing the wire-crane system. Another technique implements lifting by holding the sternum with a large clamp [10]. This idea advanced from simple hooking, by lifting with a retractor instead of human pulling. However, the access angle to the sternal margin would be vertical and difficult to grasp in deep depressions with a steeply rotated sternum. Finally, a vacuum apparatus for sucking up the chest wall depression seems to be readily applicable, but its sucking power can lift the sternum only to a limited degree and the device obstructs the view of the major part of the surgical field [2].

In comparison with other systems, the author's crane system with sternal wiring carriers has numerous advantages. First, sternal stitching has proven to be a quick and complication-free technique in thousands of attempts. Sec- ond, there is no limit to the location or the number of stitches. Third, sternal wiring can be easily repeated and leaves no scar. Fourth, the wire loops are linkable to any available table-mount retractors, including the Thompson retractor, Rultract, the pectus crane of the author's first design, or finally, the Easy Crane system (Fig. 5). Fifth, it keeps the sternum consistently lifted at the desired level throughout the procedure without undue human effort.

Wire suturing to the sternum is not difficult once the knack is acquired. The key is to stay within the matrix of the bone while passing the needle through, as the needle delivered in the right direction has virtually no chance of reaching the heart or pericardium. We proved its safety in thousands of sternal stitching procedures. Occasionally, the wire cut through the bony tissue of the sternum, but without causing any major problems, and we could readily make another stitch at a better spot next to it.

Despite its advantages, many surgeons have been uncomfortable with sternal stitching. We believed that screwing would make the crane technique easier and more adoptable than blind wire suturing. The crane system using a sternal screw is a sophisticated and future-oriented method to bring the surgical armamentarium for pectus excavatum a step forward. The screw offers advantages of additional comfort and security over sternal stitching. The screws safely engage in the sternum at a predetermined depth. The screwing location can be liberal according to the morphology of the chest wall, and multiple screws help to lift heavy chests or multiple targets to be included in the repair (Fig. 3).

The screws were designed with different threads than the usual sternal screws to tolerate higher pressure from lifting heavy chests (Fig. 2). Screws with different diameters and lengths can be selected according to the sternal thickness
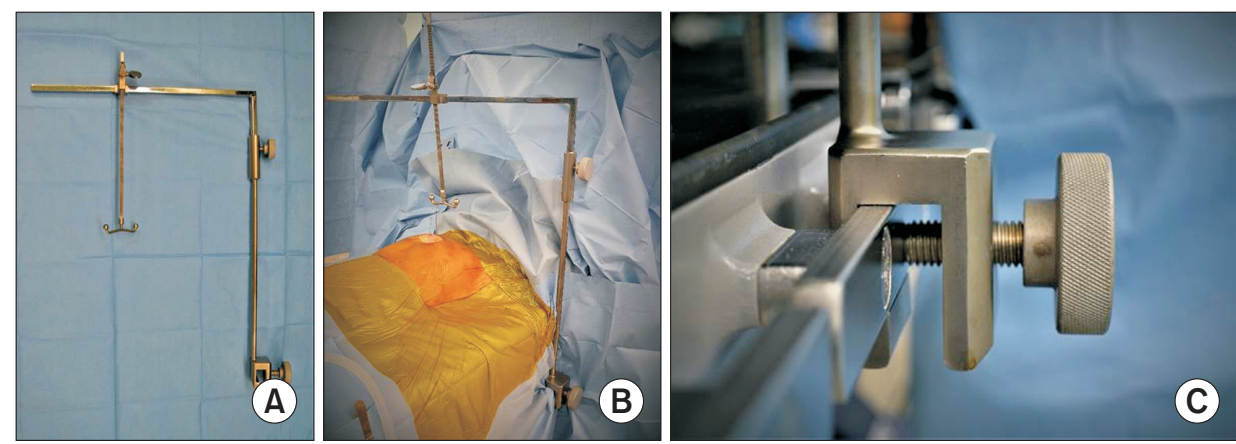

Fig. 5. The "Easy Crane" system. (A) The crane system involves a vertical pole for operating the table mount, a sidearm for connection with a wire-loop from the sternum, and ratchets to wind up the sternum. (B) Set-up of the crane with versatile directions of the side arm to follow the targets in the chest wall to lift. (C) The slot at the tip of the vertical pole is designed to easily slide onto the side rail from above. It offers easy on-and-off placement and removal of the crane system during the operation. 
and the expected weight of the chest wall. Through the torsion and pull-out tests, we proved that the screws were strong enough for chest wall lifting. The pull-out tests from the polyurethane blocks and fresh porcine sternum revealed that the strength of the screws far exceeded the weight they are expected to bear. The porcine sternal model showed that the screw sustained more than 1,200 N. Those numbers are well over the estimated sternal lifting force measured in real patients, where the forces ranged from 70 to $250 \mathrm{~N}$ [11].

In conclusion, our test data on the polyurethane blocks and the porcine sternum showed that the screws were easily driven in and effectively sustained the pressure. The screw-crane system would be feasible for sternum lifting in pectus excavatum repair procedures.

\section{Conflict of interest}

No potential conflict of interest relevant to this article was reported.

\section{ORCID}

Hyung Joo Park: https://orcid.org/0000-0003-0886-0817

Gongmin Rim: https://orcid.org/0000-0002-0982-7851

\section{References}

1. Park HJ, Jeong JY, Jo WM, et al. Minimally invasive repair of pectus excavatum: a novel morphology-tailored, patient-specific approach. J Thorac Cardiovasc Surg 2010;139:379-86.
2. Haecker FM, Krebs T, Kocher GJ, Schmid RA, Sesia SB. Sternal elevation techniques during the minimally invasive repair of pectus excavatum. Interact Cardiovasc Thorac Surg 2019;29:497-502.

3. Park HJ, Kim KS, Lee S, Jeon HW. A next-generation pectus excavatum repair technique: new devices make a difference. Ann Thorac Surg 2015;99:455-61.

4. Park HJ, Lee SY, Lee CS, Youm W, Lee KR. The Nuss procedure for pectus excavatum: evolution of techniques and early results on 322 patients. Ann Thorac Surg 2004;77:289-95.

5. Nuss D, Kelly RE Jr, Croitoru DP, Katz ME. A 10-year review of a minimally invasive technique for the correction of pectus excavatum. J Pediatr Surg 1998;33:545-52.

6. Hebra A, Kelly RE, Ferro MM, Yuksel M, Campos JR, Nuss D. Life-threatening complications and mortality of minimally invasive pectus surgery. J Pediatr Surg 2018;53:728-32.

7. Park HJ, Lee SY, Lee CS. Complications associated with the Nuss procedure: analysis of risk factors and suggested measures for prevention of complications. J Pediatr Surg 2004;39:391-5.

8. Tedde ML, de Campos JR, Wihlm JM, Jatene FB. The Nuss procedure made safer: an effective and simple sternal elevation manoeuvre. Eur J Cardiothorac Surg 2012;42:890-1.

9. Rygl M, Vyhnanek M, Kucera A, Mixa V, Kyncl M, Snajdauf J. Technical innovation in minimally invasive repair of pectus excavatum. Pediatr Surg Int 2014;30:113-7.

10. Jaroszewski DE, Johnson K, McMahon L, Notrica D. Sternal elevation before passing bars: a technique for improving visualization and facilitating minimally invasive pectus excavatum repair in adult patients. J Thorac Cardiovasc Surg 2014;147:1093-5.

11. Weber PG, Huemmer HP, Reingruber B. Forces to be overcome in correction of pectus excavatum. J Thorac Cardiovasc Surg 2006;132: 1369-73. 